\title{
Interactive Exploration of Physically-Observable Objective Vortices in Unsteady 2D Flow
}

\author{
Xingdi Zhang, Markus Hadwiger, Thomas Theußl, Peter Rautek
}

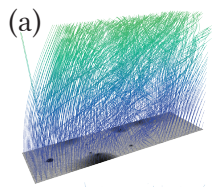

(b)

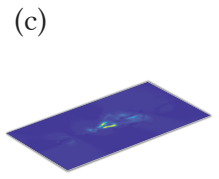

(d)

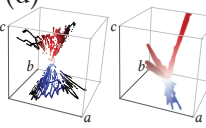

(e)

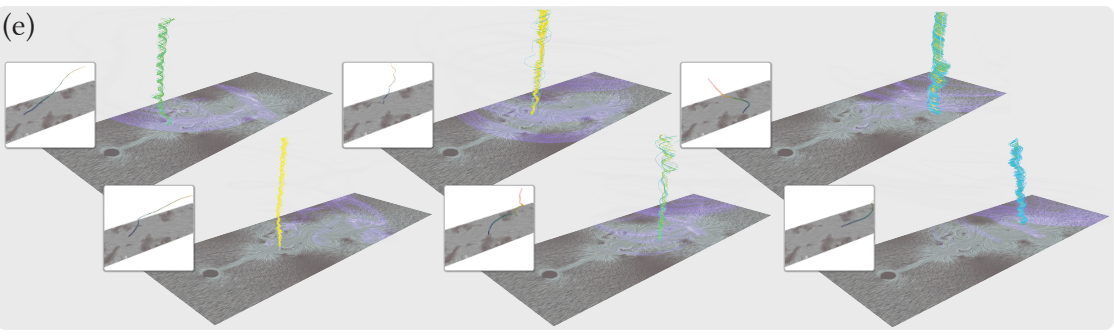

Fig. 1. Vortex structures (b; $\lambda_{2}$ vortex cores) not visible in the input flow (a) become visible relative to different observers (e). (Vertical axis is time.) Because all observers (six observers in insets) were computed using objective optimization, the observed vortices are also objective. However, each observer is also physically realizable. This guarantees that these vortices are also physically observable. We represent observers as curves through the Lie algebra of physically-realizable observer motions, which is a special 3D vector space. Choosing a basis defines an $(a, b, c)$ parameter space (d), enabling efficient averaging, interpolation, and comparison of observers (c).

Abstract-State-of-the-art computation and visualization of vortices in unsteady fluid flow employ objective vortex criteria, which makes them independent of reference frames or observers. However, objectivity by itself, although crucial, is not sufficient to guarantee that one can identify physically-realizable observers that would perceive or detect the same vortices. Moreover, a significant challenge is that a single reference frame is often not sufficient to accurately observe multiple vortices that follow different motions. This paper presents a novel framework for the exploration and use of an interactively-chosen set of observers, of the resulting relative velocity fields, and of objective vortex structures. We show that our approach facilitates the objective detection and visualization of vortices relative to well-adapted reference frame motions, while at the same time guaranteeing that these observers are in fact physically realizable. In order to represent and manipulate observers efficiently, we make use of the low-dimensional vector space structure of the Lie algebra of physically-realizable observer motions. We illustrate that our framework facilitates the efficient choice and guided exploration of objective vortices in unsteady 2D flow, on planar as well as on spherical domains, using well-adapted reference frames.

Index Terms-Flow visualization, vortex detection, objectivity, observers, reference frames, Lie algebras

\section{INTRODUCTION}

A significant amount of recent research in flow visualization and continuum mechanics has built on the concept of objectivity $[14,17,19,39]$. For vortex detection, for instance, it is crucial that the employed criteria are objective, because otherwise different observers can come to different conclusions on where vortices are located. For this reason, non-objective criteria often might not correspond to physical reality. However, even objectivity, by itself, only guarantees that different observers reach the same conclusions, but not necessarily how physicallyplausible these conclusions are. In particular, it is known that for many real-world flow phenomena a single reference frame is not sufficient in order to detect or depict all features of interest [14,29]. For this reason, generic objective approaches have been proposed that use a large number of observers and detect features jointly $[4,14,18,39]$. These approaches, however, focus on overall objectivity, and not on confirming that each detected feature would in fact be able to be perceived by a physically-realizable observer. In this paper, we therefore target the additional consideration whether some, a priori unknown, but

- Xingdi Zhang, Markus Hadwiger, and Peter Rautek are with King Abdullah University of Science and Technology (KAUST), Visual Computing Center, Thuwal, 23955-6900, Saudi Arabia. E-mail: \{xingdi.zhang, markus.hadwiger, peter.rautek\} @kaust.edu.sa.

- Thomas Theußl is with King Abdullah University of Science and Technology (KAUST), Core Labs, Thuwal, 23955-6900, Saudi Arabia.

E-mail: thomas.theussl@kaust.edu.sa.

Manuscript received 31 Mar. 2021; accepted xx xxx. 201x. Date of Publication $x x x x x .201 x$; date of current version $x x x x x$. 201x. For information on obtaining reprints of this article, please send e-mail to: reprints@ieee.org. Digital Object Identifier: $x x . x x x x / T V C G .201 x . x x x x x x x$ physically-realizable, observer is able to reach the same conclusion.

We propose to address the gap between using a single, physicallyrealizable observer on the one hand, and joint methods using many observers on the other hand, via an interactive framework for $2 \mathrm{D}$ unsteady flow that (1) employs only physically-realizable observers; and (2) enables users to explore arbitrary sets of observers together with the flow phenomena they perceive, in order to ultimately determine a set of observers that is sufficient to detect and visualize all structures of interest. A crucial property of our framework is that we enable interactively choosing, averaging, and interpolating observers, coupled with interactive observer-relative visualization and feature detection.

\subsection{Mathematical Framework}

Before introducing our framework, we first briefly introduce several crucial concepts that are not standard in the flow visualization literature. We refer to later sections as well as the appendixes in the supplementary material for details and more background on these concepts.

Physically-realizable observers. We call a reference frame that is described by a time-dependent rigid motion a physically-realizable observer, since every reference frame motion that can be carried out in reality must preserve Euclidean distances [46] (neglecting relativistic effects). The most important consequence of this fact in our context is that we can thus model all such observers by vector fields that are the derivatives of rigid motions, obtaining the motion via integration.

Killing vector fields. The derivatives of rigid motions are given by Killing vector fields [32,38], corresponding to the infinitesimal isometries of a given manifold $M$. In our context, we focus on $M$ being either the plane $\mathbb{R}^{2}$ or the two-sphere $\mathbb{S}^{2}$, defining our domain for $2 \mathrm{D}$ unsteady flow fields. Corresponding to the notion of physically-realizable observers, we can therefore describe all possible observer motions by (time-dependent) Killing vector fields. Every motion described by a 

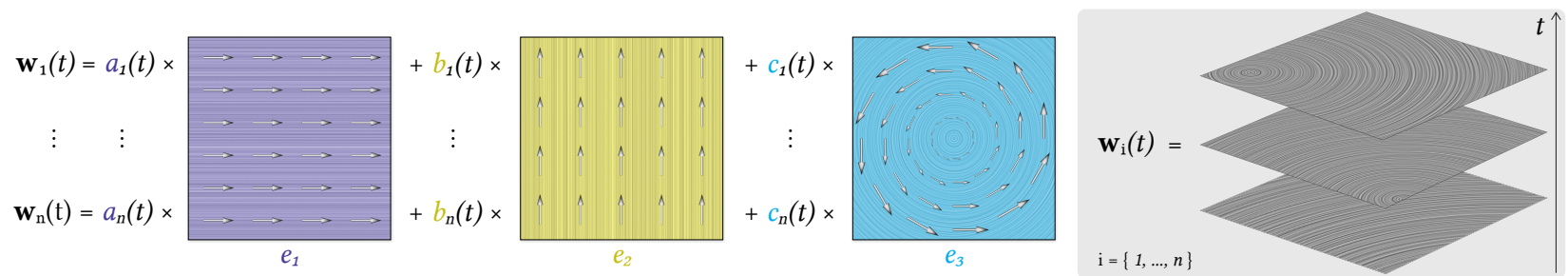

Fig. 2. Observer representation. Any physically-realizable observer is determined by a time-dependent Killing field $(x, t) \mapsto \mathbf{w}(x, t)=a(t) \mathbf{e}_{1}(x)+$ $b(t) \mathbf{e}_{2}(x)+c(t) \mathbf{e}_{3}(x)$. The basis vector fields $\mathbf{e}_{1}$ (purple), $\mathbf{e}_{2}$ (yellow), and $\mathbf{e}_{3}$ (cyan) are steady vector fields that do not change over time. Any possible (time-dependent) observer $\mathbf{w}_{i}$ is solely determined by a time-dependent function $t \mapsto\left(a_{i}(t), b_{i}(t), c_{i}(t)\right)$ of three scalar coefficients $\left(a_{i}, b_{i}, c_{i}\right)$ per time $t$.

Killing field is guaranteed to be physically realizable. Any observer that cannot be modeled by a Killing field is not physically realizable.

Lie algebra of observer motions. A Lie algebra [12] is a vector space, of abstract vector objects with scalar multiplication and vector addition, plus an additional vector product, the Lie bracket $[\cdot, \cdot]$. In our context, however, each vector (element) of a Lie algebra represents an entire vector field on the underlying manifold $M$. This enables us to represent all physically-realizable observer motions as elements of the Lie algebra of Killing fields on the manifold $M$. Most importantly, as a vector space, every Lie algebra has a basis. Therefore, any observer motion can be given as a linear combination of basis Killing fields.

Notation. We denote vectors and vector fields in bold, like $\mathbf{v}, \mathbf{u}$, and real numbers as well as spatial points in non-bold, like $a, t$, or $x \in M$.

\section{Our framework}

For both the manifolds $M=\mathbb{R}^{2}$ and $M=\mathbb{S}^{2}$, respectively, the Lie algebra of physically-realizable observer motions is three-dimensional (as a vector space). Thus, all possible observer motions are fully determined by coefficients referred to a three-dimensional basis. We choose a fixed basis of three basis Killing vector fields $\left\{\mathbf{e}_{1}, \mathbf{e}_{2}, \mathbf{e}_{3}\right\}$ on $M$, and represent every possible observer motion by three real coefficients $(a, b, c)$ for each time $t$. Any observer is therefore determined by a function

$$
t \mapsto(a(t), b(t), c(t)) .
$$

The corresponding observer motion is given by a time-dependent Killing vector field $\mathbf{w}$, determining the motion via its derivative, as

$$
(x, t) \mapsto \mathbf{w}(x, t)=a(t) \mathbf{e}_{1}(x)+b(t) \mathbf{e}_{2}(x)+c(t) \mathbf{e}_{3}(x) .
$$

See Fig. 2. This representation enables averaging, interpolating, and comparing observers simply via their coefficients given by Eq. 1 .

\subsection{Interactive Exploration Pipeline}

Fig. 3 depicts an overview of our interactive exploration framework. The goal is to explore an arbitrary, unsteady input flow field v. However, in order to do so, we want to be able to use an arbitrary reference frame, relative to which the field $\mathbf{v}$ is visualized, and features such as vortices can be computed objectively. To facilitate this, the second input to our system is another unsteady flow field $\mathbf{u}$, which is used with the semantics of an observer field [18]. We can initialize the field $\mathbf{u}$ by computing (or loading) the solution of an objective optimization [14,18,39], but we can also simply use the field $\mathbf{u}:=\mathbf{v}$. The latter can be used to "extract" observers from the input field $\mathbf{v}$ itself. Independent of how the observer field $\mathbf{u}$ is initialized, our framework represents all observers $\mathbf{w}$ by functions $t \mapsto(a(t), b(t), c(t))$ (Eq. 1), determining observers by either (1) extraction from the observer field $\mathbf{u}$; or (2) averaging or interpolation of already known observers. When an observer is chosen interactively, the input field $\mathbf{v}$ can immediately be visualized relative to it. Furthermore, we can visualize the functions $t \mapsto(a(t), b(t), c(t))$ as curves in the three-dimensional parameter space $(a, b, c) \subset \mathbb{R}^{3}$ (Fig. 3 (center)).

\subsection{Observers and Objectivity}

A fundamental property of our framework is that we determine observers objectively, in the sense that all possible observers agree on which specific reference frame motions were chosen ${ }^{1}$. This has the consequence that properties of the input field computed relative to any one

${ }^{1}$ We call these objectively-determined observers. This is not a standard term, but it captures the concept well. Appendix B provides a detailed discussion. such observer are objective. Furthermore, because all our observers are physically realizable, these properties are therefore also physically observable. Our observers are determined objectively because we "extract" them along path lines of the observer field $\mathbf{u}$, which are Lagrangian and thus objective [21], and because of a crucial theorem about Killing fields (Theorem 3.2). However, this only holds when all possible observers do, in fact, agree on the motion described by the field $\mathbf{u}$. This is trivially the case when we choose $\mathbf{u}:=\mathbf{v}$. Crucially, however, the same is true when $\mathbf{u}$ is computed via objective optimization, as those of Günther et al. [14], Hadwiger et al. [18], or Rautek et al. [39]. Furthermore, we allow determining new observers by averaging or interpolation of a set of observers. If the latter are all objectively determined, the resulting new observer is again objectively determined. For more details and some subtleties, we refer to Appendix B (supplementary material).

\subsection{Contributions}

We propose the first framework for the interactive, objective exploration of unsteady 2D flow from the perspective of a set of physicallyrealizable observers. In contrast to prior work that uses a large number of observers jointly, such as one observer per space-time grid point [14], or a continuous field of observers [18,39], we focus on facilitating visualization and feature detection with respect to individual observers.

In particular, we for the first time (1) enable the interactive selection, comparison, and user evaluation of objective vortex structures relative to an interactively-chosen and modified set of physically-realizable observers; (2) define a novel mathematical framework that enables the efficient manipulation of observers by expressing all possible observer motions in the same basis of vector fields for the corresponding Lie algebra; (3) describe how previous methods integrate with our interactive framework; and (4) leverage the Lie algebra of observer motions to treat flow fields in 2D flat space and the curved surface of a sphere, e.g., for geophysical flow fields, respectively, in a conceptually unified way.

\section{Related Work}

Flow visualization is one of the core topics of scientific visualization, and many techniques have been developed over the years to visualize flow fields, for example LIC for steady (time-independent) flow fields [8], or texture advection methods for unsteady (time-dependent) flow fields [25,47], as well as the visualization of integral curves [26,49]. Instead of flow visualization in general, in this section we focus on related work whose specific aspects are most relevant to our work.

Reference frames. Flow fields are velocity vector fields, and therefore they can only be given with respect to a frame of reference, or an observer. One important area where this fact is important is in the detection and visualization of vortices, where it has become a major consideration when evaluating different vortex detection methods $[17,19]$. A crucial notion is that of the invariance of a given method with respect to a particular kind of reference frame transformation, for example Galilean invariance [48], rotation invariance [15], or invariance relative to rigid motions [19]. The latter is known as objectivity [19] or frame indifference [46], originally defined in continuum mechanics $[22,33,46]$, and recognized to be of fundamental importance for vortex detection more recently $[14,17,19,21]$, although used earlier as well $[1,11]$.

Multiple reference frames. Most commonly, only a single reference frame is considered for flow visualization or computational tasks such as vortex detection. Several recent methods, however, jointly consider multiple reference frames, such as averaging observers related to a 


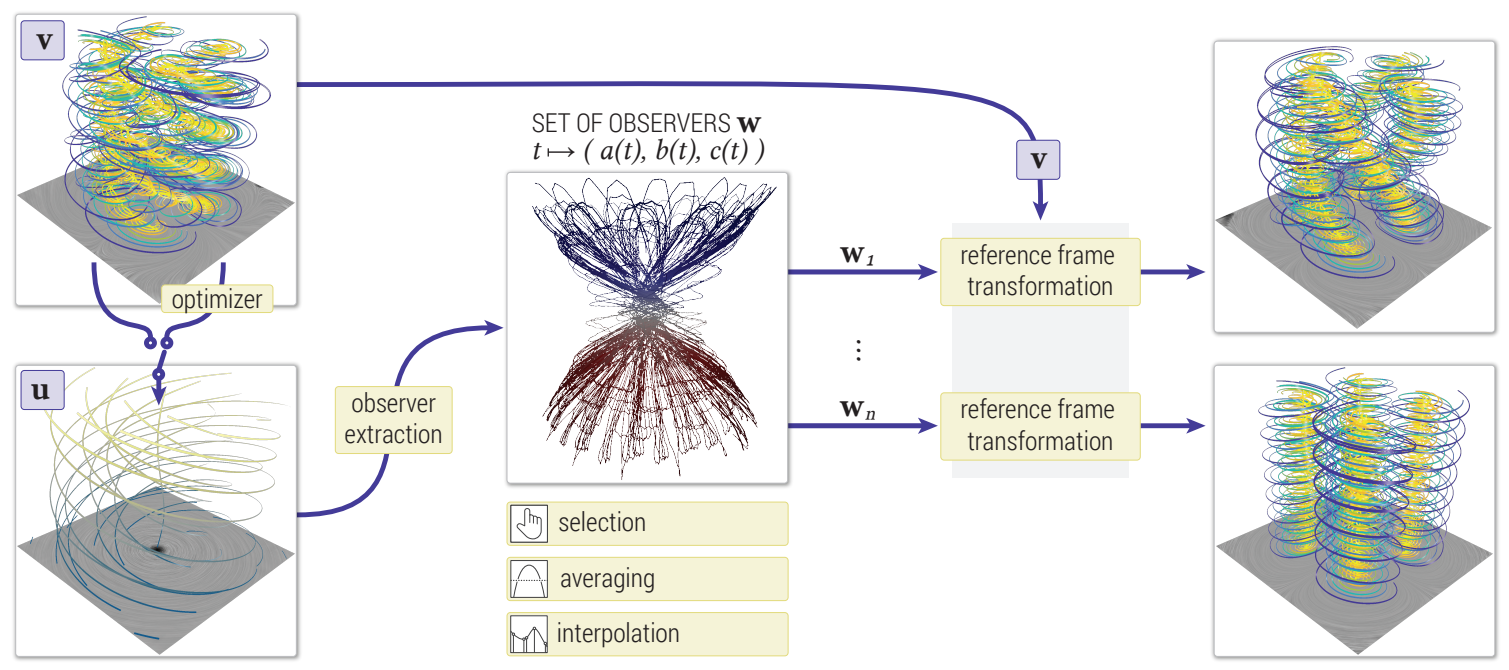

Fig. 3. Interactive exploration pipeline. The input to our system consists of two unsteady vector fields: the input flow field $\mathbf{v}$, and an observer field $\mathbf{u}$. The observer field can be computed via objective optimization [14,18], or can be initialized to $\mathbf{u}:=\mathbf{v}$ (to extract observers from the input field). Observers are extracted from the field $\mathbf{u}$, representing each corresponding Killing field $\mathbf{w}$ by a function $t \mapsto(a(t), b(t), c(t))$. New observers can be generated by averaging or interpolation. When an observer is selected, the input field $\mathbf{v}$ can be visualized interactively relative to that observer.

finite set of Galilean-invariant critical points [7], one observer per point in space-time [14], or a continuous field of observers [18,39].

Vortex detection. Vortices are important features in flow visualization [17] and fluid mechanics [41]. There is no single, well-defined notion of a vortex, but they are usually seen as a swirling motion around a central region $[1,10,29,40]$. Earlier methods have often used only spatial (in contrast to time) derivatives, e.g., the well-known methods of Okubo [34] and Weiss [50], Sujudi and Haimes [44], the Q Criterion of Hunt et al. [23], or the $\lambda_{2}$ criterion of Jeong and Hussein [24], which can lead to challenges for unsteady flow. One reason for this is that many early methods are partially based on critical point theory of steady vector fields $[36,37]$. Later extensions were improved for unsteady flow, e.g., with Galilean invariance $[42,48]$, often building on the parallel vectors operator [35] or a predictor-corrector approach [5]. Criteria can be grouped into line-based vs. region-based techniques [17], the latter often including the detection of region boundaries [13,21]. The objectivity of vortex criteria is now seen to be a crucial desired property of vortex detectors $[14,17,19,21]$. One particular recent example that we also use is the Lagrangian-Averaged Vorticity Deviation (LAVD) [21].

Generic objectivization. A recent idea for developing vortex detection methods that are objective is to solve an optimization problem that enables generically "objectivizing" non-objective vortex criteria $[4,14,16,18,39]$. All of these approaches perform objective computations that define a large number of observers. The motivation for this is the well-established understanding that for many flow fields a single observer is not able to depict all vortices of interest [29]. However, while the objectivity of these approaches guarantees that any physically-realizable, i.e., rigidly-moving, observer will reach the same conclusions regarding the "joint" detection of vortices, none of these methods guarantees that there is one physically-realizable observer that by itself (i.e., not jointly) would have computed the same vortex using physically-motivated criteria. While this does not change the fact that the above methods are provably objective $[14,18,39,45]$, recent work has argued that physical criteria should also be used to determine the validity and consistency of vortex detection [20]. In this work, we approach this problem from a different perspective to try and combine the advantages of both viewpoints: We enable the interactive exploration of multiple, objectively-determined observers, but allow users to choose specific, physically-realizable observers for actual vortex detection and visualization. In this way, physically-motivated criteria fully apply.

Killing vector fields. The properties of Killing fields are often explored in Riemannian geometry $[9,32,38]$. They have been used explicitly in geometry $[6,28,31,43]$, including vector field design on curved surfaces [2,3], and recently also in flow visualization $[18,39]$.

\section{Observers and Sets of Observers}

Our approach is based on a set of physically-realizable observers. In this section, we describe how these observers are modeled as Killing vector fields, and how the corresponding description can be extracted from any given input observer velocity field $\mathbf{u}$ (Fig. 3, bottom left).

\subsection{Physically-Realizable Observers}

The central basic notion of this paper is given by the following

Theorem 3.1. An observer is physically realizable if its time-dependent motion relative to any other physically-realizable observer is a rigid motion, i.e., if the map between the two reference frames is an isometry.

This basically means that every rigid reference frame motion corresponds to a physically-realizable observer. The reason for the "circular" argument above is that all motion is relative, and thus for a rigid motion to in fact describe a physical observer, the background reference frame (e.g., the lab frame) must already be known to be physically realizable. In terms of our input field $\mathbf{v}$, this means that the whole field must have been measured relative to the same, physically-realizable observer.

\section{Killing vector fields}

A vector field $x \mapsto \mathbf{w}(x)$ is a Killing field, if its (spatial) velocity gradient $\nabla \mathbf{w}$ is identically anti-symmetric. That is, when for $\mathbf{w}$ we have

$$
\langle\nabla \mathbf{w}(\mathbf{x}), \mathbf{x}\rangle=0
$$

This must hold at all points $x \in M$, for all vectors $\mathbf{x}$. Eq. 3 is valid for all manifolds $M$, with $\nabla \mathbf{w}$ the covariant derivative of $\mathbf{w}$ (in components, $\nabla_{j} w^{i}$; see Rautek et al. [39] for details). For $M=\mathbb{R}^{2}$ with Cartesian coordinates, $\nabla \mathbf{w}$ is simply a Jacobian matrix $\partial_{j} w^{i}$ of partial derivatives.

\section{Time-dependent Killing fields}

We define a time-dependent Killing field with the properties given above to hold for each fixed time $t$, giving a time-dependent field

$$
(x, t) \mapsto \mathbf{w}(x, t) .
$$

Corollary 3.1. A time-dependent Killing field $\mathbf{w}(x, t)$ gives the derivative of a time-dependent rigid motion. Therefore, it corresponds to a physically-realizable observer, defined by integrating the Killing field.

As above, it is important that $\mathbf{w}$ is measured relative to a physicallyrealizable observer. We will also exploit the following important

Theorem 3.2. A Killing field $\mathbf{w}$ is uniquely defined by: (1) a single vector $\mathbf{w}(x)$ at an arbitrarily chosen point $x \in M$, and (2) the corresponding (anti-symmetric) velocity gradient $(\nabla \mathbf{w})_{x}$ at the same point $x$. 

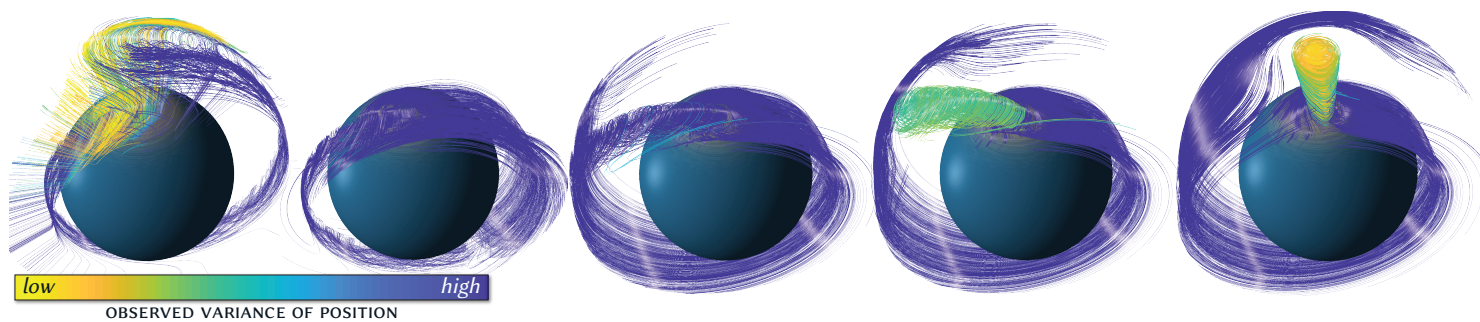

Fig. 4. Vortex on the sphere. We exploit the same 3D vector space structure of the Lie algebra of observer motions on spherical domains as in the plane. Here, we smoothly interpolate between two observers, the input lab frame (left) and an objectively-determined observer [39] (right), smoothly "shifting" the observed field and the contained vortex until the latter becomes visible (right). (The axis orthogonal to the sphere corresponds to time.)

This is a well-known property of Killing fields [38, p.315]. However, this property is crucial to objectively "extracting" physically-realizable observers from the methods described in prior work [14, 18,39], and converting them to the representation of our framework. See Sec. 3.3.

\subsection{The Lie Algebra of Observer Motions}

We exploit that the Lie algebras of Killing fields on our manifolds $M$ of interest, the plane $\mathbb{R}^{2}$ and the sphere $\mathbb{S}^{2}$, are three-dimensional vector spaces, and thus have 3D vector space bases. (See also Appendix A.)

\section{Dimensionality and basis expansion}

It is crucial to note that the Lie algebra of Killing fields is a vector space of vector fields, i.e., each element (abstract vector) of this vector space is itself a whole vector field on the underlying manifold $M$. It is also in this sense that the Lie algebras that we use are three-dimensional: Each Killing field can be referred to a basis comprising three basis vector fields, each weighted by a scalar coefficient. We have the following

Theorem 3.3. A Killing field $\mathbf{w}$ on a manifold $M$ is uniquely defined by a spanning set of basis Killing fields $\left\{\mathbf{e}_{i}\right\}$ and the corresponding set of coefficients $\left\{w^{i}\right\}$. The Killing field $\mathbf{w}$ is then given as $\mathbf{w}=\sum_{i} w^{i} \mathbf{e}_{i}$.

In this paper, all vector spaces of Killing fields are three-dimensional, and we will therefore only need three coefficients $w^{i}$, which we will instead denote by $a:=w^{1}, b:=w^{2}, c:=w^{3}$, or collectively by $(a, b, c)$.

\section{Inner products of vector fields}

To be able to determine orthogonality of basis functions as well as for comparing observers by comparing their corresponding Killing fields, we will make use of an inner product between vector fields, defined on the Lie algebra of observer motions. We define the inner product between two (Killing) vector fields $\mathbf{w}_{1}$ and $\mathbf{w}_{2}$, with domain $D$, by

$$
\left\langle\left\langle\mathbf{w}_{1}, \mathbf{w}_{2}\right\rangle\right\rangle:=\int_{D}\left\langle\mathbf{w}_{1}, \mathbf{w}_{2}\right\rangle_{x} d A .
$$

Here, the usual inner product in the tangent space at each point $x \in D$ is denoted by $\langle\cdot, \cdot\rangle_{x}$, and $d A$ denotes integration over area elements. This inner product is defined with respect to a domain $D \subseteq M$. For $M=\mathbb{S}^{2}$, we use $D=\mathbb{S}^{2}$, because $\mathbb{S}^{2}$ is compact and the integral is therefore guaranteed to be finite. For $M=\mathbb{R}^{2}$, we typically integrate over a compact rectangular subdomain $D=\left[x_{a}, x_{b}\right] \times\left[y_{a}, y_{b}\right] \subset \mathbb{R}^{2}$.

\section{Efficient computation of inner products}

Instead of computing the above integrals for every arbitrary pair of observers $\left(\mathbf{w}_{1}, \mathbf{w}_{2}\right)$, we can exploit the vector space structure of the Lie algebra of Killing fields. From this structure, we know that every Killing field can be written as a linear combination $\mathbf{w}=\sum_{i} w^{i} \mathbf{e}_{i}$, where the $\mathbf{e}_{i}$ are basis Killing vector fields on the manifold $M$. Using the inner product $\langle\langle\cdot, \cdot\rangle\rangle$ between two vector fields given by Eq. 5, we can define a metric tensor $g_{i j}$ of Killing vector fields, given by the components

$$
g_{i j}:=\left\langle\left\langle\mathbf{e}_{i}, \mathbf{e}_{j}\right\rangle\right\rangle .
$$

Given this metric tensor, we can then compute the inner product between any two Killing fields simply from their components as

$$
\left\langle\left\langle\mathbf{w}_{1}, \mathbf{w}_{2}\right\rangle\right\rangle=\sum_{i, j} g_{i j} w_{1}^{i} w_{2}^{j} .
$$

For more details, we refer to Appendix C (supplementary material).

\section{Basis Killing fields in the Euclidean plane}

The Lie algebra of Killing fields on $M=\mathbb{R}^{2}$ is three-dimensional, and we construct the following three linearly-independent basis Killing fields in $\mathbb{R}^{2}$, where the vectors at any point $x=(\hat{x}, \hat{y}) \in \mathbb{R}^{2}$ are

$$
\mathbf{e}_{1}(\hat{x}, \hat{y})=\left[\begin{array}{l}
1 \\
0
\end{array}\right], \mathbf{e}_{2}(\hat{x}, \hat{y})=\left[\begin{array}{l}
0 \\
1
\end{array}\right], \mathbf{e}_{3}(\hat{x}, \hat{y})=\left[\begin{array}{cc}
0 & -1 \\
1 & 0
\end{array}\right]\left[\begin{array}{l}
\bar{x} \\
\bar{y}
\end{array}\right] .
$$

See Fig. 2. For the field $\mathbf{e}_{3}$, corresponding to $\mathbf{v}$ being given on a rectangular domain $D=\left[x_{a}, x_{b}\right] \times\left[y_{a}, y_{b}\right] \subset \mathbb{R}^{2}$, with center point $\left(\hat{x}_{0}, \hat{y}_{0}\right)=$ $\frac{1}{2}\left(x_{a}+x_{b}, y_{a}+y_{b}\right)$, we have defined $(\bar{x}, \bar{y}):=\left(\hat{x}-\hat{x}_{0}, \hat{y}-\hat{y}_{0}\right)$.

Each basis element must be a Killing field. To confirm, we compute

$$
\nabla \mathbf{e}_{1}=0, \quad \nabla \mathbf{e}_{2}=0, \quad \nabla \mathbf{e}_{3}=\left[\begin{array}{cc}
0 & -1 \\
1 & 0
\end{array}\right] .
$$

Therefore, Eq. 3 indeed holds for all fields $\mathbf{e}_{i}$, i.e., we have

$$
\left\langle\nabla \mathbf{e}_{i}(\mathbf{x}), \mathbf{x}\right\rangle=0,
$$

for all $i \in\{1,2,3\}$, and for all vectors $\mathbf{x}$, at all points $x \in M=\mathbb{R}^{2}$.

Using this basis, we can therefore write any Killing field $\mathbf{w}$ on $M=$ $\mathbb{R}^{2}$ as $\mathbf{w}=a \mathbf{e}_{1}+b \mathbf{e}_{2}+c \mathbf{e}_{3}$, with three coefficients $(a, b, c)$. We note that, due to this particular choice of basis, $(a, b)$ have the meaning of a linear velocity vector (given with two Cartesian components), and the third coefficient $c$ has the meaning of angular velocity. ${ }^{2}$ In fact, the linear velocity is the same constant vector at all points $x \in \mathbb{R}^{2}$.

Orthogonality. Due to symmetry, this basis is orthogonal for a rectangular domain of integration with $\left(\hat{x}_{0}, \hat{y}_{0}\right)$ chosen in the center, where, in particular, we have $\left\langle\left\langle\mathbf{e}_{1}, \mathbf{e}_{3}\right\rangle\right\rangle=\left\langle\left\langle\mathbf{e}_{2}, \mathbf{e}_{3}\right\rangle\right\rangle=0$, because $\left\langle\mathbf{e}_{1}(\bar{x}, \bar{y}), \mathbf{e}_{3}(\bar{x}, \bar{y})\right\rangle=-\left\langle\mathbf{e}_{1}(-\bar{x},-\bar{y}), \mathbf{e}_{3}(-\bar{x},-\bar{y})\right\rangle$, and likewise for the fields $\mathbf{e}_{2}$ and $\mathbf{e}_{3}$. The orthogonality $\left\langle\left\langle\mathbf{e}_{1}, \mathbf{e}_{2}\right\rangle\right\rangle=0$ immediately follows from the orthogonality $\left\langle\mathbf{e}_{1}(\hat{x}, \hat{y}), \mathbf{e}_{2}(\hat{x}, \hat{y})\right\rangle=0$ at every point $(\hat{x}, \hat{y})$.

See Eq. 5 for the definition of the vector field inner product $\langle\langle\cdot, \cdot\rangle\rangle$.

\section{Basis Killing fields on the sphere}

The Lie algebra of Killing fields on $M=\mathbb{S}^{2}$ is also three-dimensional. We construct the following three basis Killing fields for the two-sphere $\mathbb{S}^{2}:=\left\{(\hat{x}, \hat{y}, \hat{z}) \mid \hat{x}^{2}+\hat{y}^{2}+\hat{z}^{2}=1\right\}$ embedded in $\mathbb{R}^{3}$, where the vectors at any point $x=(\hat{x}, \hat{y}, \hat{z})$, as elements of the tangent space embedded in $\mathbb{R}^{3}$ at that point, are given by (see Fig. 10 in the supplementary material)

$$
\begin{aligned}
& \mathbf{e}_{1}(\hat{x}, \hat{y}, \hat{z})=\left[\begin{array}{ccc}
0 & 0 & 0 \\
0 & 0 & -1 \\
0 & 1 & 0
\end{array}\right]\left[\begin{array}{l}
\hat{x} \\
\hat{y} \\
\hat{z}
\end{array}\right], \mathbf{e}_{3}(\hat{x}, \hat{y}, \hat{z})=\left[\begin{array}{ccc}
0 & -1 & 0 \\
1 & 0 & 0 \\
0 & 0 & 0
\end{array}\right]\left[\begin{array}{c}
\hat{x} \\
\hat{y} \\
\hat{z}
\end{array}\right], \\
& \mathbf{e}_{2}(\hat{x}, \hat{y}, \hat{z})=\left[\begin{array}{ccc}
0 & 0 & 1 \\
0 & 0 & 0 \\
-1 & 0 & 0
\end{array}\right]\left[\begin{array}{l}
\hat{x} \\
\hat{y} \\
\hat{z}
\end{array}\right] .
\end{aligned}
$$

Using this basis, we can write any Killing field $\mathbf{w}$ on $M=\mathbb{S}^{2}$ as $\mathbf{w}=$ $a \mathbf{e}_{1}+b \mathbf{e}_{2}+c \mathbf{e}_{3}$, with three coefficients $(a, b, c)$. We note that, due to the above choice of basis, the coefficients $(a, b, c)$ determine a 3D angular velocity vector, and $\omega^{2}:=a^{2}+b^{2}+c^{2}$ is the corresponding

\footnotetext{
${ }^{2}$ As for any vector space, other bases are of course also possible. Here then, however, no single coefficient would be the angular velocity. See Appendix A.
} 

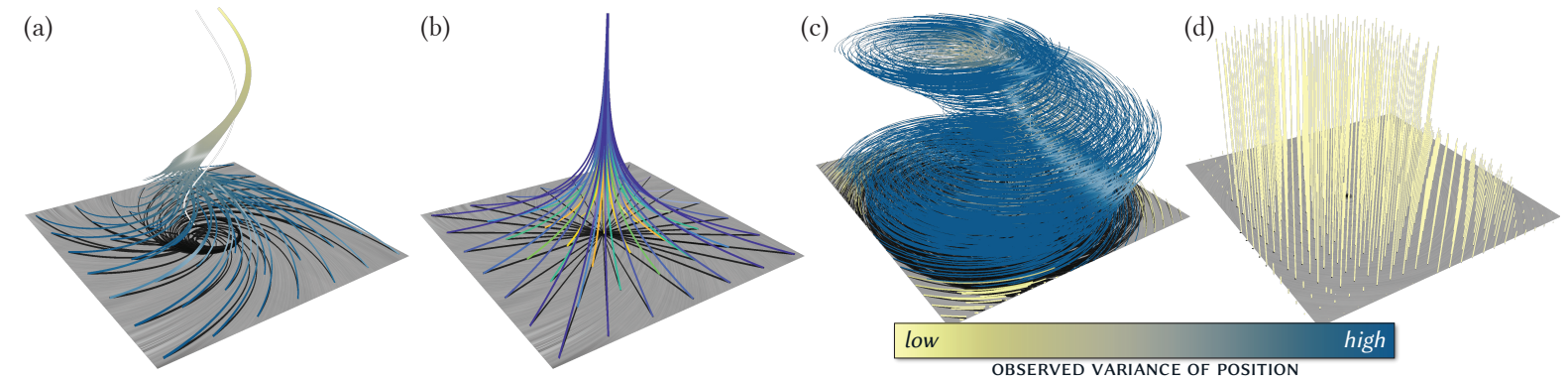

Fig. 5. Beads flow. (Original; a, b) The input flow field (vertical axis is time) contains rotational as well as contracting motion (negative divergence). However, using our framework one can determine that all rotational parts of this field can be fully explained by a rotating reference frame. (Divergencefree; c, d) Observer exploration enables us to see that, in fact, this flow field is itself a Killing field. Thus, the input field (c) can be explained purely by a moving reference frame observing a 0-field (d). This indicates that this field does not contain any intrinsic motion, beyond the motion of an observer.

(squared) angular velocity (magnitude). Since Eq. 3 is intrinsically defined in each tangent space, to see that the $2 \mathrm{D}$ tensors $\nabla \mathbf{e}_{i}$ are antisymmetric, we must compute the covariant derivatives $\nabla \mathbf{e}_{i}$ at all $x \in \mathbb{S}^{2}$. Using a right-handed orthonormal basis in each tangent plane, they are

$$
\left(\nabla \mathbf{e}_{i}\right)_{x}=\left[\begin{array}{cc}
0 & -\cos \varphi_{i}(x) \\
\cos \varphi_{i}(x) & 0
\end{array}\right] .
$$

Here, the angle $\varphi_{i}(x) \in[0, \pi]$ is the colatitude of $x$ away from the "north pole" of the respective $\mathbf{e}_{i}$, i.e., $\hat{x}=1$ for $\mathbf{e}_{1}, \hat{y}=1$ for $\mathbf{e}_{2}, \hat{z}=1$ for $\mathbf{e}_{3}$. Therefore, Eq. 3 again holds for all $\mathbf{e}_{i}$, i.e., $\left\langle\nabla \mathbf{e}_{i}(\mathbf{x}), \mathbf{x}\right\rangle=0$ for all $\mathbf{x}$.

Orthogonality. This basis is also orthogonal, i.e., $\left\langle\left\langle\mathbf{e}_{i}, \mathbf{e}_{j}\right\rangle\right\rangle=0$ for all $i \neq j$. This can be confirmed by analytic integration over the sphere.

\section{Representation of observers}

We define an observer as the time-dependent, rigid reference frame motion given by a time-dependent Killing field $\mathbf{w}$. Instead of storing an explicit vector field $(x, t) \mapsto \mathbf{w}(x, t)$ for each observer, we store observers with respect to a chosen basis of Killing fields. While in principle this basis is arbitrary, we use the basis vector fields given by Eq. 8 (for Euclidean domains $M=\mathbb{R}^{2}$ ), and Eq. 11 (for spherical domains $M=\mathbb{S}^{2}$ ), respectively. Any given observer $\mathbf{w}$ is thus given by a function $t \mapsto((a(t), b(t), c(t))$ (Eq. 1), which at any time allows us to obtain the corresponding time-dependent Killing field w from Eq. 2.

\section{Comparing observers}

We exploit the low-dimensional (in our case, three-dimensional) vector space structure of Killing vector fields in order to efficiently quantify the similarity of two observers given as time-dependent Killing fields.

First, from the definition of the inner product of two vector fields given by Eq. 5, we can define the difference between two arbitrary (time-independent) Killing fields as the scalar-valued distance function

$$
d\left(\mathbf{w}_{1}, \mathbf{w}_{2}\right):=\sqrt{\left\langle\left\langle\mathbf{w}_{1}-\mathbf{w}_{2}, \mathbf{w}_{1}-\mathbf{w}_{2}\right\rangle\right\rangle} .
$$

This definition in fact defines a metric on the vector space of all Killing fields in the domain $D \subseteq M$, where we want to compare observers. For time-dependent Killing fields $\mathbf{w}_{1}(x, t)$ and $\mathbf{w}_{2}(x, t)$, we now define

$$
d\left(\mathbf{w}_{1}(t), \mathbf{w}_{2}(t) ; t_{0}, t_{1}\right):=\int_{t_{0}}^{t_{1}} d\left(\mathbf{w}_{1}(t), \mathbf{w}_{2}(t)\right) d t .
$$

Here, we have integrated over a desired time window $\left[t_{0}, t_{1}\right]$, over which we want to compare the two observers. The rationale for this definition is simply that we integrate the per-time distance values over a time window of interest, in order to quantify the "total" difference between the two observers over that time window. (Longer time windows, in general, give larger distances, when the two fields are different.)

We note that when both $\mathbf{w}_{1}$ and $\mathbf{w}_{2}$ are objectively-determined observers, then both distance functions above give objective scalars.

\subsection{Determination of Observer Sets}

We can store a set of observers by storing multiple observers, each represented by a function $t \mapsto(a(t), b(t), c(t))$, as above (Eq. 1).

However, we can also "extract" an arbitrary number of observers from a given vector field, which we will refer to as the observer field $\mathbf{u}$.

\section{Observer world lines}

Theorem 3.2 shows that an observer can also be specified by a function

$$
t \mapsto\left(x(t), \mathbf{w}(x(t), t),(\nabla \mathbf{w})_{(x(t), t)}\right),
$$

where $t \mapsto x(t)$ is an arbitrary path on the manifold $M$, and we prescribe the corresponding vectors $\mathbf{w}$, and tensors $\nabla \mathbf{w}$ along this path. Although compared to Eq. 1, the above representation requires more storage, this way of specifying Killing fields enables the following general approach for extracting observers from a given observer velocity field $\mathbf{u}$.

\section{Observer world lines from observer fields}

It is possible to avoid storing the path $t \mapsto x(t)$ in the previous definition by defining it as the path line of a vector field $\mathbf{u}(x(t), t)$ given along the path $x(t)$, which is obtained by solving the non-autonomous ODE

$$
\frac{d}{d t} x(t)=\mathbf{u}(x(t), t)
$$

with an initial value $x\left(t_{0}\right)$, i.e., some chosen position on the path line $t \mapsto x(t)$ at time $t=t_{0}$, for some chosen time $t_{0}$. We call this path $t \mapsto$ $x(t)$ a world line [18] of the corresponding observer. In order to extract an observer, at each time $t$ we refer to the corresponding point $x(t)$.

\section{Extraction from observer field in the Euclidean plane}

Given a single vector $\mathbf{u}(x)$ and the velocity gradient $(\nabla \mathbf{u})_{x}$ at any point $x \in M=\mathbb{R}^{2}$, we can extract the coefficients of the corresponding Killing field w, with respect to the basis fields $\left\{\mathbf{e}_{1}, \mathbf{e}_{2}, \mathbf{e}_{3}\right\}$ (Eq. 8), as

$$
\nabla \mathbf{w}=\frac{1}{2}\left((\nabla \mathbf{u})_{x}-(\nabla \mathbf{u})_{x}^{T}\right)=\left[\begin{array}{cc}
0 & -c \\
c & 0
\end{array}\right], \mathbf{u}(x)+\nabla \mathbf{w}(\mathbf{r})=\left[\begin{array}{l}
a \\
b
\end{array}\right]
$$

We define $\mathbf{r}:=\left(\hat{x}_{0}-\hat{x}, \hat{y}_{0}-\hat{y}\right)^{T}$, with $(\hat{x}, \hat{y})$ the Cartesian coordinates of the point $x$, and $\left(\hat{x}_{0}, \hat{y}_{0}\right)$ the center point used in the definition of the basis vector field $\mathbf{e}_{3}$. The coefficients $(a, b, c)$ now determine the Killing field $\mathbf{w}=a \mathbf{e}_{1}+b \mathbf{e}_{2}+c \mathbf{e}_{3}$, referred to the basis given in Eq. 8 .

\section{Extraction from observer field on the sphere}

Given a single vector $\mathbf{u}(x)$ and the covariant derivative $(\nabla \mathbf{u})_{x}$ at any point $x \in M=\mathbb{S}^{2}$, we can extract the coefficients of the corresponding Killing field $\mathbf{w}$, with respect to the basis fields $\left\{\mathbf{e}_{1}, \mathbf{e}_{2}, \mathbf{e}_{3}\right\}$ (Eq. 11), using the following approach. Referring to the point $x \in \mathbb{S}^{2}$ as the position vector $\mathbf{r}(x):=(\hat{x}, \hat{y}, \hat{z})^{T}$ in Cartesian coordinates, we can define the following orthonormal basis, written as column vectors of the matrix

$$
\hat{\mathbf{B}}:=[\mathbf{x}|\mathbf{z} \times \mathbf{x}| \mathbf{z}], \quad \mathbf{x}:=\mathbf{u}(x) /\|\mathbf{u}(x)\|, \quad \mathbf{z}:=\mathbf{r}(x) .
$$

When $\|\mathbf{u}(x)\|=0, \mathbf{x}$ can be any unit vector in the tangent plane at $x$. Given the covariant derivative $\nabla \mathbf{u}$ in components as $\nabla_{j} u^{i}$ in some coordinate chart with tangent space basis $\left\{\mathbf{b}_{1}, \mathbf{b}_{2}\right\}$ at $x$, and the coordinate 

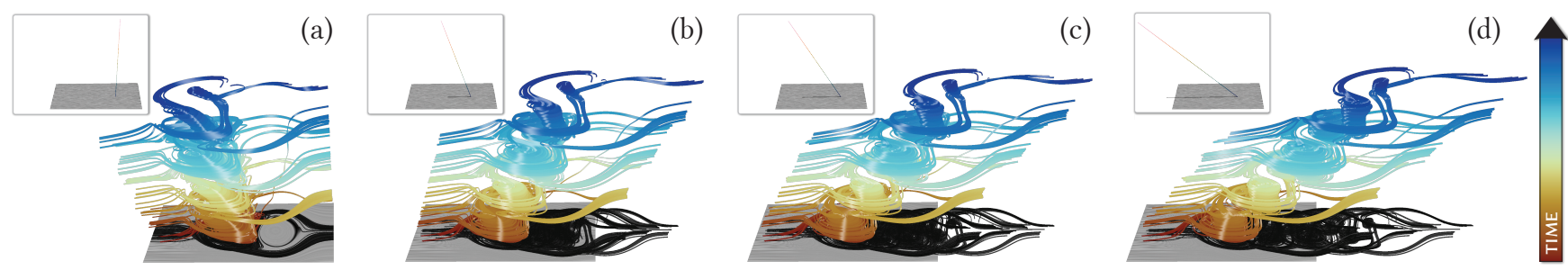

Fig. 6. Cylinder flow. The vortex structures in this data set are short-lived. They appear and disappear frequently over time. In the input lab frame (a) different vortices overlap (over time) around the same spatial position and appear to be the same vortex. We interpolate (a-d) between the lab frame and an objectively-determined constant-velocity observer that "comoves" with the vortices, revealing them more clearly. The insets depict observer world lines. Time corresponds to the vertical axis and is also color-coded to help highlight how individual vortices appear and disappear over time.

Jacobian $\mathbf{J}$ transforming from that basis to the basis $\hat{\mathbf{B}}$, we can compute ${ }^{3}$

$$
\nabla_{j} \hat{u}^{i}=\mathbf{J}\left(\nabla_{j} u^{i}\right) \mathbf{J}^{-1}, \quad \nabla_{j} \hat{w}^{i}=\frac{1}{2}\left(\nabla_{j} \hat{u}^{i}-\nabla_{i} \hat{u}^{j}\right)=\left[\begin{array}{cc}
0 & -\hat{c} \\
\hat{c} & 0
\end{array}\right] .
$$

Given the chart tangent space basis $\left\{\mathbf{b}_{1}, \mathbf{b}_{2}\right\}$, embedded in $\mathbb{R}^{3}$, the Jacobian $\mathbf{J}$ is given by the top-left $2 \times 2$ submatrix of the $3 \times 3$ matrix

$$
\hat{\mathbf{J}}=\hat{\mathbf{B}}^{T}\left[\mathbf{b}_{1}\left|\mathbf{b}_{2}\right| \mathbf{z}\right]
$$

From the obtained $\hat{c}$, and the length $\hat{b}:=\|\mathbf{u}(x)\|$, we can now compute

$$
\hat{\mathbf{K}}=\left[\begin{array}{rrr}
0 & -\hat{c} & \hat{b} \\
\hat{c} & 0 & 0 \\
-\hat{b} & 0 & 0
\end{array}\right], \quad \mathbf{K}=\hat{\mathbf{B}} \hat{\mathbf{K}} \hat{\mathbf{B}}^{T}=\left[\begin{array}{rrr}
0 & -c & b \\
c & 0 & -a \\
-b & a & 0
\end{array}\right]
$$

The coefficients $(a, b, c)$ obtained from the matrix $\mathbf{K}$ now determine the Killing field $\mathbf{w}=a \mathbf{e}_{1}+b \mathbf{e}_{2}+c \mathbf{e}_{3}$, referred to the basis given in Eq. 11

For completeness, we note that here $\hat{b}=\omega \sin \varphi$ and $\hat{c}=\omega \cos \varphi$, where $\omega^{2}=\hat{b}^{2}+\hat{c}^{2}=a^{2}+b^{2}+c^{2}$ is the (squared) angular velocity of the global rotation of the sphere given by $\mathbf{w}$, with $\varphi$ the colatitude of $x$ relative to the critical point of $\mathbf{w}$ that is the "north pole" of the rotation, i.e., the point $(\bar{x}, \bar{y}, \bar{z})^{T}=(a, b, c)^{T} /\left\|(a, b, c)^{T}\right\|$, defined when $\omega \neq 0$.

\section{Observer Averaging and INTERPolation}

Using the vector space structure of all observer Killing fields, we can directly average a set of observers and interpolate between observers.

\subsection{Observer Averaging}

Given a set of observers $\left\{\mathbf{w}_{i}\right\}_{i \in I}$ with the index $i \in I$ from some index set $I$, considering the vector space structure of the space of all observers, we can simply define the average observer of this set of observers as

$$
t \mapsto(\bar{a}(t), \bar{b}(t), \bar{c}(t)):=\frac{1}{|I|}\left(\sum_{i \in I} a_{i}(t), \sum_{i \in I} b_{i}(t), \sum_{i \in I} c_{i}(t)\right),
$$

where $|I|$ is the cardinality of the index set $I$. If $I$ is a whole region of a manifold $M$ instead of a discrete set, we define the above average with integrals instead of sums, and the cardinality for normalization by an appropriate measure of the set $I$, e.g., the area measure on $M$.

Sec. 6.3 describes an application of this averaging technique, applied over a region $I=U(t) \subset \mathbb{R}^{2}$, with the standard area measure on $\mathbb{R}^{2}$.

\subsection{Direct Observer Interpolation (Blending)}

Given two observers $\mathbf{w}_{1}=a_{1} \mathbf{e}_{1}+b_{1} \mathbf{e}_{2}+c_{1} \mathbf{e}_{3}$ and $\mathbf{w}_{2}=a_{2} \mathbf{e}_{1}+b_{2} \mathbf{e}_{2}+$ $c_{2} \mathbf{e}_{3}$, we can directly interpolate between them via $(\alpha \in[0,1])$

$$
\begin{aligned}
\mathbf{w}(t ; \alpha)= & \left((1-\alpha) a_{1}(t)+\alpha a_{2}(t)\right) \mathbf{e}_{1}+ \\
& \left((1-\alpha) b_{1}(t)+\alpha b_{2}(t)\right) \mathbf{e}_{2}+ \\
& \left((1-\alpha) c_{1}(t)+\alpha c_{2}(t)\right) \mathbf{e}_{3}
\end{aligned}
$$

${ }^{3}$ We note that the anti-symmetrization used here depends on the fact that the basis $\hat{\mathbf{B}}$ is orthonormal, and hence the corresponding metric tensor is the identity. Otherwise, $\nabla_{j} u_{i}$ and the metric $g_{i j}$ would have to be used explicitly. See [39].
Due to the linearity of the Lie algebra of Killing fields, this works for both $M=\mathbb{R}^{2}$ and $M=\mathbb{S}^{2}$, by using the corresponding basis $\left\{\mathbf{e}_{i}\right\}$.

See Fig. 6 for an example, where we directly interpolate between the (input) lab frame observer (given by the observer field $\mathbf{u}=0$ ) and an observer "comoving" along with the vortices. (However, see Appendix B for additional discussion on using the lab frame observer.)

\subsection{Observer Interpolation Along a Path}

Given an arbitrary observer field $\mathbf{u}$, we can pick two points $x_{1}(t) \in M$ and $x_{2}(t) \in M$, respectively, for an arbitrarily chosen fixed time $t$. We can then extract observers corresponding to any interpolated point $x(t ; \alpha) \in M$, in between the two points. For simplicity, for $M=\mathbb{R}^{2}$ we connect the two points by a line segment, obtaining

$$
x(t ; \alpha)=(1-\alpha) x_{1}(t)+\alpha x_{2}(t), \quad 0 \leq \alpha \leq 1 .
$$

For $M=\mathbb{S}^{2}$, the linear connection between the two points does not exist on the sphere, and we therefore use the geodesic arc between $x_{1}(t)$ and $x_{2}(t)$ instead, again interpolating according to $\alpha \in[0,1]$.

See the accompanying paper video for examples.

\section{Observer-Relative Quantities}

We want to be able to interactively compute the observed input vector field $\mathbf{v}$ and its derivatives, relative to the rigid motion described by $\mathbf{w}$. In terms of the observer $\mathbf{w}$ given by the function $t \mapsto(a(t), b(t), c(t))$, below we will make use of the following expansions and derivatives,

$$
\begin{aligned}
\mathbf{w}(x, t) & =a(t) \mathbf{e}_{1}(x)+b(t) \mathbf{e}_{2}(x)+c(t) \mathbf{e}_{3}(x), \\
\nabla \mathbf{w}(x, t) & =a(t) \nabla \mathbf{e}_{1}(x)+b(t) \nabla \mathbf{e}_{2}(x)+c(t) \nabla \mathbf{e}_{3}(x), \\
\frac{\partial \mathbf{w}(x, t)}{\partial t} & =\frac{d a(t)}{d t} \mathbf{e}_{1}(x)+\frac{d b(t)}{d t} \mathbf{e}_{2}(x)+\frac{d c(t)}{d t} \mathbf{e}_{3}(x) .
\end{aligned}
$$

\subsection{Reference Frame Transformation}

In order to transform between two reference frames, we define a time-dependent diffeomorphism $t \mapsto \phi_{t}$, with each diffeomorphism, for fixed $t$, mapping from $M$ to $M$, i.e., $\phi_{t}: M \rightarrow M$. For physicallyrealizable observers, each diffeomorphism $\phi_{t}$ is in fact an isometry.

For $M=\mathbb{R}^{2}$, the isometry $\phi_{t}$ can be written explicitly as

$$
\phi_{t}(x)=w(t)+\mathbf{Q}(t)\left(x-w\left(t_{0}\right)\right),
$$

where $w\left(t_{0}\right)$ is some arbitrary position at $t=t_{0}$, and each $\mathbf{Q}(t)$ is a rotation tensor. In Cartesian coordinates, the rotation $\mathbf{Q}(t)$ is given by

$$
\mathbf{Q}(t)=\left[\begin{array}{rr}
\cos \theta(t) & -\sin \theta(t) \\
\sin \theta(t) & \cos \theta(t)
\end{array}\right]
$$

The path $t \mapsto w(t)$ and the integrated angle $\theta(t)$ are the solutions of

$$
\frac{d}{d t} w(t)=\mathbf{w}(w(t), t), \quad \frac{d}{d t} \boldsymbol{\theta}(t)=c(t),
$$

with $\theta\left(t_{0}\right)=0$. We can solve these two ODEs explicitly by

$$
w(t)=w\left(t_{0}\right)+\int_{t_{0}}^{t} \mathbf{w}(w(\tau), \tau) d \tau, \quad \theta(t)=\int_{t_{0}}^{t} c(\tau) d \tau .
$$



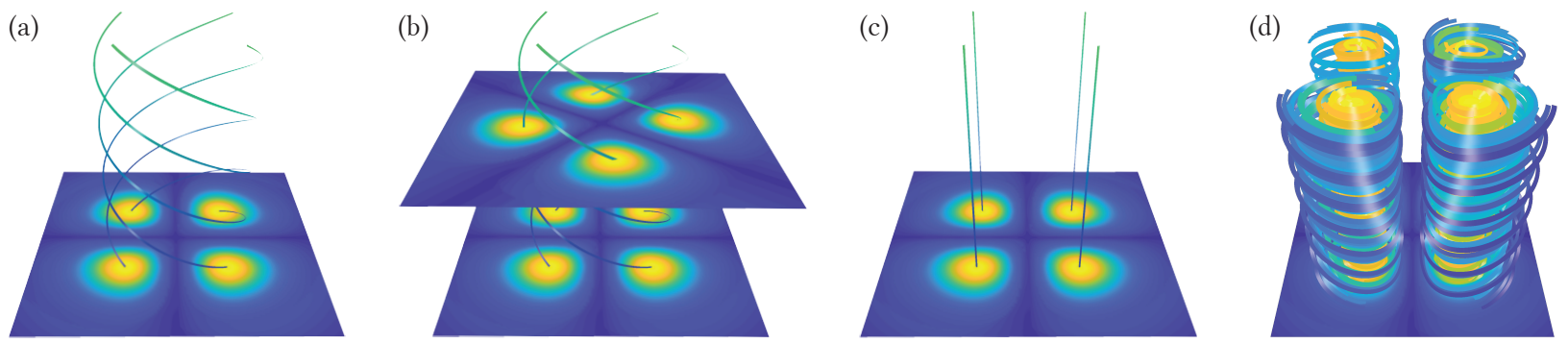

Fig. 7. Four centers. (a,b) LAVD [21] and $\lambda_{2}$ vortex core lines agree (vertical axis is time). (c) Vortex core lines observed relative to an observer w selected from an observer field $\mathbf{u}$ computed via optimization [18]. (d) Observed path lines swirling around the core lines using variance color-coding.

To transform vectors and tensors with the diffeomorphism $\phi_{t}$, we use the pullback $\phi_{t}^{*}$. On $M=\mathbb{R}^{2}$, the pullback $\phi_{t}^{*} \mathbf{x}$ of a vector field $\mathbf{x}$ is

$$
\phi_{t}^{*} \mathbf{x}=\mathbf{Q}^{T}(t) \mathbf{x} .
$$

The pullback $\phi_{t}^{*} \mathbf{T}$ of a second-order tensor field $\mathbf{T}$ (a linear map) is

$$
\phi_{t}^{*} \mathbf{T}=\mathbf{Q}^{T}(t) \mathbf{T} \mathbf{Q}(t) .
$$

For details on diffeomorphisms and pullbacks, and explicit formulas for $M=\mathbb{S}^{2}$, we refer to Appendixes D and E (supplementary material).

\subsection{Observed Velocity}

The observed velocity, with respect to the observer $\mathbf{w}$, is obtained as

$$
\mathbf{v}^{*}=\phi_{t}^{*}(\mathbf{v}-\mathbf{w}) .
$$

This velocity is of particular importance for vortex detectors that search for critical points of the velocity field, i.e., in this case the critical points of $\mathbf{v}^{*}$. In terms of the observer $\mathbf{w}$ as $t \mapsto(a(t), b(t), c(t))$, this becomes

$$
\mathbf{v}^{*}=\phi_{t}^{*}\left(\mathbf{v}-a(t) \mathbf{e}_{1}-b(t) \mathbf{e}_{2}-c(t) \mathbf{e}_{3}\right) .
$$

\subsection{Observed Velocity Gradient}

The observed velocity gradient, with respect to the observer $\mathbf{w}$, is

$$
\nabla \mathbf{v}^{*}=\phi_{t}^{*}(\nabla \mathbf{v}-\nabla \mathbf{w}) .
$$

This velocity gradient is of particular importance for all vortex detectors that compute its eigenvalues and determine vortex-like behavior only when there are complex eigenvalues (2D: one conjugate complex pair). For these criteria, the relevant eigenvalues are therefore those of $\nabla \mathbf{v}^{*}$.

In terms of the observer $\mathbf{w}$ as $t \mapsto(a(t), b(t), c(t))$, this becomes

$$
\nabla \mathbf{v}^{*}=\phi_{t}^{*}\left(\nabla \mathbf{v}-a(t) \nabla \mathbf{e}_{1}-b(t) \nabla \mathbf{e}_{2}-c(t) \nabla \mathbf{e}_{3}\right) .
$$

\subsection{Observed Time Derivative}

The observed time derivative $[18,39]$, with respect to the observer $\mathbf{w}$, is

$$
\begin{aligned}
\frac{\partial \mathbf{v}^{*}}{\partial t} & =\phi_{t}^{*}\left(\frac{\mathscr{D}}{\mathscr{D} t}(\mathbf{v}-\mathbf{w})\right) \\
& =\phi_{t}^{*}\left(\frac{\partial \mathbf{v}}{\partial t}-\frac{\partial \mathbf{w}}{\partial t}+\nabla \mathbf{v}(\mathbf{w})-\nabla \mathbf{w}(\mathbf{v})\right) .
\end{aligned}
$$

In terms of the observer $\mathbf{w}$ as $t \mapsto(a(t), b(t), c(t))$, this becomes

$$
\begin{array}{r}
\frac{\partial \mathbf{v}^{*}}{\partial t}=\phi_{t}^{*}\left(\frac{\partial \mathbf{v}}{\partial t}-\frac{d a(t)}{d t} \mathbf{e}_{1}-\frac{d b(t)}{d t} \mathbf{e}_{2}-\frac{d c(t)}{d t} \mathbf{e}_{3}+\right. \\
\nabla \mathbf{v}\left(a(t) \mathbf{e}_{1}+b(t) \mathbf{e}_{2}+c(t) \mathbf{e}_{3}\right)- \\
\left.\left(a(t) \nabla \mathbf{e}_{1}+b(t) \nabla \mathbf{e}_{2}+c(t) \nabla \mathbf{e}_{3}\right) \mathbf{v}\right) .
\end{array}
$$

See Appendix F (supplementary material) and previous work [18, 39] for more on observed time derivatives. In addition, we give equations for the observed acceleration in Appendix G (supplementary material).

\section{Determining Observers from Prior Methods}

We now demonstrate the generality and versatility of our framework by describing how observers can be defined via (1) Extraction from a globally optimized, continuous field of observers [18,39]; (2) Extraction from separate observers optimized at each space-time point [14]; (3) Replication of Lagrangian-Averaged Vorticity Deviation (LAVD) [21] in our framework, relative to a special objectively-determined observer.

\subsection{Observer Fields from Global Optimization}

A directly suitable choice for the observer field $\mathbf{u}$ required by our framework are the observer fields computed by global optimization methods in the plane [18], or on the sphere [39], respectively. We have used this approach in the results shown in Fig. 4, Fig. 7, and Fig. 9.

\subsection{Observer Fields from Generic Objective Vortices}

In order to obtain a valid observer field from the generic objective vortices method of Günther et al. [14], we first use their optimization method to compute an optimal, objective field $\overline{\mathbf{v}}$ (Eq. 20 in [14]). From the objective field $\overline{\mathbf{v}}$, we then compute a smooth observer field $\mathbf{u}$ as

$$
\mathbf{u}:=\mathbf{v}-\overline{\mathbf{v}}
$$

This simple approach allows us to directly use the objective optimization method of Günther et al. [14] as an input to our framework. We have computed the observer field used in Fig. 1 with this approach.

We note that the above approach is objective and can indeed be used to obtain a valid observer field $\mathbf{u}$, despite the fact that the optimization [14] computes a different observer for each point in space-time. Since the objective vector field $\overline{\mathbf{v}}$ is defined at each space-time point, the observer field obtained via Eq. 38 describes valid, smooth observer motions. In fact, these motions are determined objectively, because the vector $\overline{\mathbf{v}}$ at each point in space-time is objective [14], and thus the corresponding velocities $\mathbf{u}$ are determined objectively. The obtained field $\mathbf{u}$ then enables the extraction of observers using our framework. We note, however, that, in general, we do not extract the exact same observers that are used for vortex detection in [14], because the derivatives given in [14] do not correspond to the field $\mathbf{u}$ that we obtain via Eq. 38.

\subsection{Observers for Lagrangian-Averaged Vorticity Deviation}

Our framework can be used to compute results equivalent to the LAVD method by Haller et al. [21], in the following way. See Fig. 7.

We define an observer $\mathbf{w}$, for $M=\mathbb{R}^{2}$, via $t \mapsto(a(t), b(t), c(t))$, by setting $a(t)=b(t)=0$, and defining $c(t):=\bar{\omega}(t)$. Here, we set the angular velocity $\bar{\omega}(t)$ to half the average vorticity magnitude ${ }^{4}$ of a chosen region $U(t) \subset \mathbb{R}^{2}$ of the flow field $\mathbf{v}$, as defined by Haller et al. [21]. As Haller et al., we assume that the region $U(t)$ is invariant under the flow of $\mathbf{v}$, i.e., the region is deformed over time by the flow (map) of $\mathbf{v}$. We can then obtain the same scalar field as the LAVD field defined by Haller et al. [21], by simply integrating the observed vorticity magnitude, as observed by the observer $\mathbf{w}$, along path lines of the field $\mathbf{v}$ in the same region $U(t)$ used for computing $\bar{\omega}(t)$. We can

${ }^{4}$ We note that the different definitions of angular velocity, vorticity (curl), and the vorticity tensor, require divisions or multiplications by two for conversion. 
obtain the observed vorticity from the observed velocity gradient $\nabla \mathbf{v}^{*}$ (Sec. 5.3), given by Eqs. 34, 35, giving the observed vorticity tensor as

$$
\mathbf{\Omega}^{*}=\frac{1}{2}\left(\nabla \mathbf{v}^{*}-\left(\nabla \mathbf{v}^{*}\right)^{T}\right) .
$$

We note that due to our definition of the observer $\mathbf{w}$, the observed vorticity magnitude is identical to the instantaneous vorticity deviation (IVD) of Haller et al. [21], and therefore its integral along path lines is identical to LAVD. However, we do not need to explicitly compute the deviation of vorticity from the average vorticity, as Haller et al. [21] do: The vorticity that we integrate is simply the regular vorticity magnitude observed by w. Nevertheless, the resulting LAVD scalar field is the same, because the observer $\mathbf{w}$ is rotating with the average angular velocity $\bar{\omega}(t)$, as defined by Haller et al. [21]. In this way, we gain additional insight into the meaning of LAVD: It is, in fact, the (integrated) vorticity magnitude seen by a particular, objectively-determined observer.

Averaging observers. We can gain even more insight from the following formulation: Given the region $U(t)$, we extract an observer from the field $\mathbf{u}:=\mathbf{v}$ at every point $x \in U(t)$, for fixed $t$, obtaining a function $t \mapsto\left(a_{i}(t), b_{i}(t), c_{i}(t)\right)$ for each $i=x$. We then define the average observer $\overline{\mathbf{w}}$ over $U(t)$ via Eq. 22 , i.e., we average $\left(a_{i}, b_{i}, c_{i}\right)$, over all $i=x \in U(t)$ to obtain $t \mapsto(\bar{a}(t), \bar{b}(t), \bar{c}(t))$. If we project this average observer to $t \mapsto(0,0, \bar{c}(t))$, this is the same observer that we defined above via the average angular velocity $\bar{\omega}(t)$. IVD and LAVD can thus be formulated using the average observer of the region $U(t)$, followed by an appropriate projection. For our basis fields $\left\{\mathbf{e}_{1}, \mathbf{e}_{2}, \mathbf{e}_{3}\right\}$, $\bar{c} \mathbf{e}_{3}$ contains the entire vorticity. We can thus either project to $\mathbf{e}_{3}$, or notice that Eq. 39 is, in fact, independent of $\mathbf{e}_{1}$ and $\mathbf{e}_{2}$ (see Eq. 9).

\section{Implementation and Performance}

We have implemented our framework for interactive observer-relative visualization in $\mathrm{C}++$ and OpenGL.

\subsection{Reference Frame Transformation}

There are two major ways for the transformation of path lines of a vector field from a given observer to another observer:

1. Transform the vector field, using Eq. 32, and integrate path lines using the standard approach in the transformed vector field.

2. Transform the geometry (vertices) of path lines computed for one observer to another observer, directly using the frame transformation given by the time-dependent diffeomorphism $\phi_{t}$.

For best interactivity, for path lines we use the second approach above:

We first compute a set of path lines for the lab frame observer, storing each path line as a vertex sequence $\left[p_{1}, p_{2}, \ldots, p_{k}\right]$. In order to display the same path lines relative to any other observer, we simply individually transform the spatial position of each vertex: For each vertex $p_{i}$, we obtain the transformed vertex via the inverse of the diffeomorphism $\phi_{t}$ given by Eq. 26, i.e.,

$$
p_{i}^{*}=\phi_{t}^{-1}\left(p_{i}\right), \text { with } t=t(i) .
$$

For $M=\mathbb{R}^{2}$, this is simply applying a rotation and a translation, for each given time $t$. However, since each vertex $p_{i}$ on the path line corresponds to a different time $t$, each vertex must be transformed with the map $\phi_{t}^{-1}$ for a different time parameter $t$, which here we refer to as $t(i)$, i.e., the time parameter corresponding to vertex number $i$. See Fig. 8 for an example for 1,200 path lines seeded on a regular grid.

For $M=\mathbb{S}^{2}$, we do the same with the map $\phi_{t}$ given in Appendix E.

\subsection{Performance}

Table 1 shows performance on a dual Intel Xeon 6230R (52 cores) and an NVIDIA GeForce RTX 3090. We first integrate path lines for the lab frame on the CPU, parallelized over all CPU cores, and then store the resulting vertex array on the GPU. Table 1 (left) shows computation times and overall throughput of vertices computed per second. When integrating enough path lines in parallel we achieve more than $15 \mathrm{M}$ vertices per second. For each time step, we typically compute two to
Table 1. Performance. Left half: path line integration, vertex throughput. Right half: observer world line integration, sample throughput.

\begin{tabular}{c||c|c|c||c|c|c} 
data set & $\begin{array}{c}\text { \# path } \\
\text { lines }\end{array}$ & $\begin{array}{c}\text { time } \\
{[\mathrm{ms}]}\end{array}$ & $\begin{array}{c}\text { vtxs } \\
\text { per s }\end{array}$ & $\begin{array}{c}\text { \# point } \\
\text { samples }\end{array}$ & $\begin{array}{c}\text { time } \\
{[\mathrm{ms}]}\end{array}$ & $\begin{array}{c}\text { samples } \\
\text { per s }\end{array}$ \\
\hline Four centers & 64 & 26 & $15 \mathrm{M}$ & $6.28 \times 10^{2}$ & 6 & $100 \mathrm{~K}$ \\
& 256 & 83 & $19 \mathrm{M}$ & $6.28 \times 10^{3}$ & 12 & $509 \mathrm{~K}$ \\
& 1,024 & 238 & $27 \mathrm{M}$ & $6.28 \times 10^{4}$ & 87 & $723 \mathrm{~K}$ \\
\hline Boussinesq & 64 & 23 & $14 \mathrm{M}$ & $5 \times 10^{2}$ & 13 & $38 \mathrm{~K}$ \\
& 256 & 76 & $17 \mathrm{M}$ & $5 \times 10^{3}$ & 21 & $234 \mathrm{~K}$ \\
& 1,024 & 293 & $17 \mathrm{M}$ & $5 \times 10^{4}$ & 82 & $609 \mathrm{~K}$ \\
\hline
\end{tabular}

ten vertices. Every time the observer changes, we compute Eq. 29 on the CPU, transfer the result to the GPU, and apply Eq. 40 to each vertex in a vertex shader. Table 1 (right) shows the throughput of samples along the observer world line. Overall, our system is fully interactive and achieves 20-30 frames per second for observed path line rendering. Performance mainly depends on the number of computed path lines and the corresponding number of vertices, and not on the data set. See Appendix $\mathrm{H}$ and $\mathrm{I}$ in the supplementary material for more details.

\section{Results}

We demonstrate different aspects of our framework on analytically defined test cases, and on numerically-simulated flow fields.

Cylinder flow (Fig. 6). The vortices behind a cylinder [27] in this data set are short-lived, and new vortices develop periodically. We demonstrate that a moving observer helps to produce a less obstructed view of the individual vortices, using an observer given by a constant velocity (translation). The vertical axis and the color map encode time in this example. By animating the observer's translation, we provide a visual clue how the data observed in the lab frame is transformed into the frame of a moving observer. The transition between the lab frame and the final observer results from direct interpolation (Sec. 4.2).

Vortex street (cylinder) (Fig. 8). This numerical simulation of a vortex street behind a cylindrical obstacle is an example for a flow field that is easier to interpret in a moving reference frame. Unlike other examples, here we have not computed an observer, but show that simply extracting an observer from the ambient flow of the input field $\mathbf{v}$ is enough to define a suitable observer. Fig. 8 shows snapshots of the interpolation between lab frame visualization (top) to the comoving observer (bottom). We point out that, although the observer was extracted along a path line in the input flow, our framework guarantees that each individual frame of the sequence corresponds to a physicallyobservable visualization, relative to a physically-realizable observer.
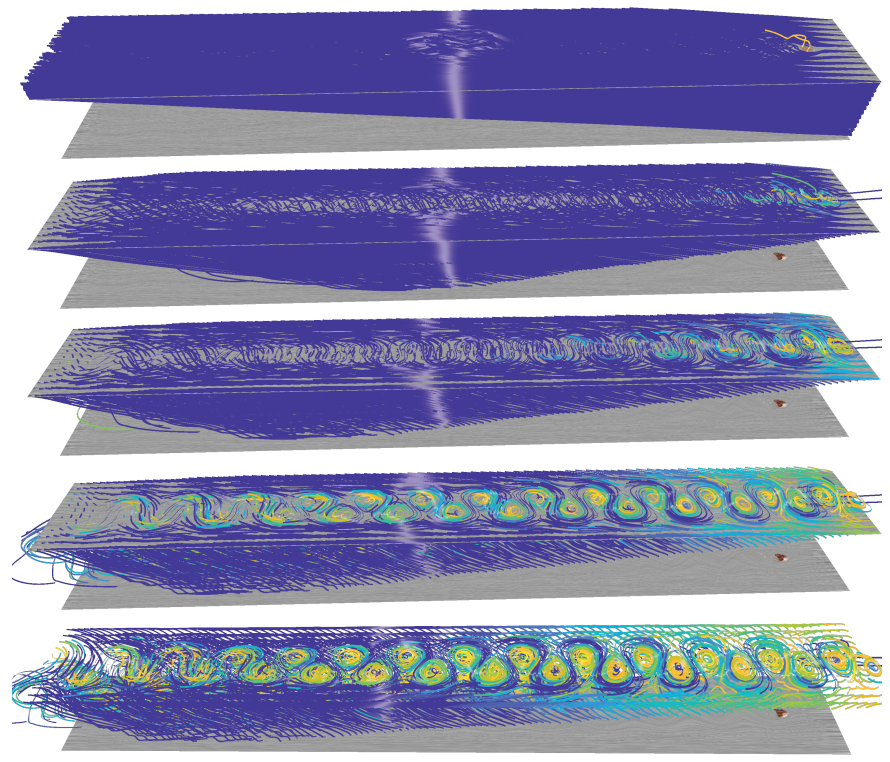

Fig. 8. Vortex street (cylinder). Changing the observer interactively (top to bottom) allows revealing the vortices (bottom) obstructed in the lab frame (top). (The vertical axis in each visualization corresponds to time.) 
We automatically compute a color-coding based on the variance of the spatial locations (relative to the chosen observer) of a particle over time, along its path line. This highlights the centers of vortices, provided that the chosen observer perceives the particle as swirling around a mostly static center. Low variance is mapped to brighter hues in the color map. This effect is clearly visible in Fig. 8 (bottom).

Bickley jet (Fig. 9). This data set [21] contains an analyticallydefined vortex street. Relative to a moving observer, the vortex structures become clearly visible. We compute the observer field $\mathbf{u}$ via global optimization [18]. Fig. 9(a) shows path lines in the lab frame, Fig. 9(b) shows observed path lines. We keep the color for each path line constant (minimum observed variance) for the transformation sequence (Fig. 9(a) to (b)). This demonstrates that color-coding is not enough to show the vortex structures. The transformation of the pathlines achieves a better visualization.

Four centers (Fig. 7). This data set by Günther et al. [15] contains four centers that rotate around a common axis. We show that LAVD [21] (color-coded) detects these four vortices, and compare LAVD with vortex core lines computed with the $\lambda_{2}$ criterion [24]. Figs. 7(a,b) show that LAVD and the $\lambda_{2}$ vortex cores agree. Fig. 7(c) shows the observed vortex core lines relative to an observer that rotates along with the vortices. We have computed this observer via global optimization [18]: The observed vortex core lines stay in place. In this reference frame, it is now trivial to verify that path lines seeded around the centers swirl around the vortex cores (Fig. 7(d)). The color coding of the swirling path lines in Fig. 7(d) is computed according to path line variance.

Beads flow (Fig. 5(a,b)). This flow was originally given by Wiebel et al. [51]. We use the analytic version used by Weinkauf and Theisel [49] and by Günther et al. [15]. It contains a contracting flow (a sink) that rotates around a center as observed by the input lab frame. Fig. 5(a) shows the input flow field, relative to the lab frame. Fig. 5(b) shows the flow relative to an observer that rotates with constant angular velocity, computed via optimization [18]. We see that all observed path lines are now straight lines (depicted as "shadows") that converge at the same point. Moreover, using our framework we have found that all observers extracted from the input field $\mathbf{v}$ perceive this flow field without any rotational motion. They are, in fact, all rotating with the same angular velocity. Since we have objectively determined observers that perceive this flow without any rotational motion, we must conclude that all observed rotation in this flow is solely due to the rotation of a reference frame, and the flow itself does not contain intrinsic rotational motion. (We note, however, that this is a purely kinematic judgement. In terms of dynamics, a rotating frame and an inertial frame behave differently.)

Divergence-free beads flow (Fig. 5(c,d)). This field is a modification of the original beads flow to make it divergence-free, used by Günther et al. [15] as a test case for rotation-invariant vortex detection. Using interactive observer exploration, we can see that the input flow in Fig. 5(c) is in fact itself a Killing field: We choose $\mathbf{u}:=\mathbf{v}$, and interactively see that all observers extracted from this observer field are in fact the same observer, as can also be confirmed via Eq. 14, and that the observer-relative visualization shows no motion at all. The observed field is identically zero, as shown in Fig. 5(d). We view this

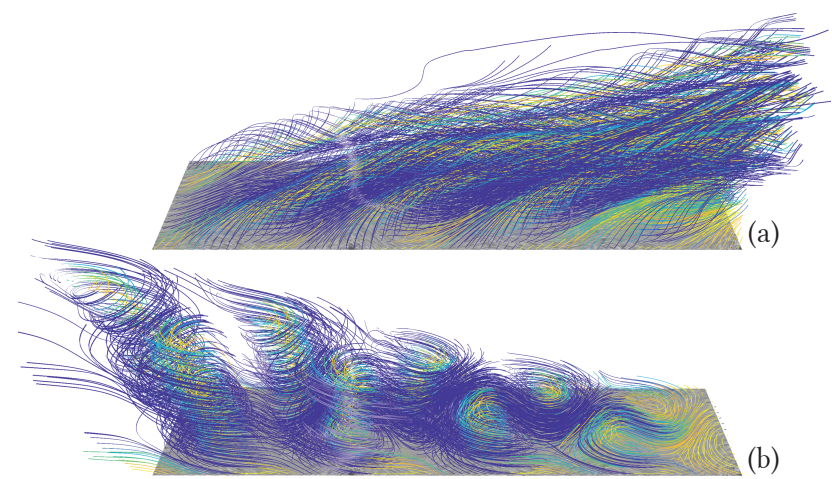

Fig. 9. Bickley jet. The input lab frame obstructs the visibility of vortices (a). A globally optimized observer [18] reveals the vortices clearly (b). (The vertical axis in each visualization corresponds to time.) field as an example of "if the motion described by a vector field is purely an observer motion, then the input field contains no intrinsic motion." The same is confirmed by an observer field computed via global optimization [18]: The optimized observer field $\mathbf{u}$ is $\mathbf{u}=\mathbf{v}$.

A fundamental problem with detecting a vortex in this data set is that, since the field can be observed as the 0-field, any rotating observer can be used in order to perceive a vortex at any chosen location. We suggest that the only consistent interpretation is that there is in fact no objective vortex contained in this field, unless one chooses to detect vortices with respect to a non-objectively chosen, special observer.

Vortex on the sphere (Fig. 4). To demonstrate that our framework is also applicable to spherical domains, we illustrate a result for a flow field with a synthetic vortex moving once around a sphere. Path lines observed relative to the lab frame are hard to interpret since they move once around the sphere, as shown in Fig. 4 (left). From left to right, Fig. 4 shows snapshots from the observer transformation between the lab frame and a globally optimized observer [39] that moves with the vortex. The last frame in the sequence clearly shows the "steady" vortex that is observed by the objectively-determined, moving observer.

Boussinesq (Fig. 1). This is a more complex scenario that requires interactive exploration of the input flow field shown in Fig. 1(a). We first use the objective optimization method by Günther et al. [14], obtaining an observer field as described in Sec. 6.2. We then visualize the flow relative to one physically-realizable observer at a time, but smoothly transition between a large number of observers: As shown in the accompanying video, we interactively move the location where an observer is extracted from the observer field. Fig. 1(e) depicts six different observer-relative visualizations. We modulate the opacity of each path line with its variance; path lines with high variance become fully transparent, making vortex structures fade in and out while the observer is being changed, as demonstrated in the video.

Figs. 1(b-d) show tools to help guide the exploration of the vast space of possible observers. Fig. 1(b) shows possible vortex core positions computed via the $\lambda_{2}$ criterion, which can help in finding good observers. Fig. 1(c) depicts color-coded observer similarity (Eq. 14), which can help identify observers that are significantly different from the current observer. Fig. 1(d) shows the $(a, b, c)$ parameter space of linear velocity (horizontal) and vorticity (vertical; positive in red, negative in blue). This provides further insight into the space of possible observers: Each observer is a curve in the 3D parameter space (left subimage); the six observers from Fig. 1(e) correspond to six curves (right subimage).

\section{Conclusions}

Our framework demonstrates that in order to reveal vortex structures in flow fields where one reference frame cannot depict all features simultaneously, interactive exploration of the space of observers is beneficial or might even be necessary. Our framework is flexible enough to incorporate observers that are objectively computed with several different available state-of-the-art methods. In contrast to prior work computing features jointly, we focus on individual, physically-realizable observers for visualization and vortex detection. We believe that our framework can provide a common basis for the future investigation of the interaction between feature detectors derived using physical arguments on the one hand, and the flexible definition of observer fields on the other.

Determining observers objectively can be crucial. For example, our exploration of the beads flow has shown that when observers are not chosen objectively, the perceived rotation can be due solely to the choice of observer: Rotation can be detected where there is no intrinsic (kinematic) rotation. However, we have also illustrated several different ways of choosing observers objectively. We therefore believe that further investigation of the meaning and implications of objective vortex detection and objectively determining observers is necessary, and we hope that our framework can contribute in this endeavor.

\section{ACKNOWLEDGMENTS}

We thank Anna Frühstïck for help with the figures and the video. This work was supported by King Abdullah University of Science and Technology (KAUST). This research used resources of the Core Labs of King Abdullah University of Science and Technology. 


\section{References}

[1] G. Astarita. Objective and generally applicable criteria for flow classification. Journal of Non-Newtonian Fluid Mechanics, 6(1):69-76, 1979.

[2] O. Azencot, M. Ben-Chen, F. Chazal, and M. Ovsjanikov. An operator approach to tangent vector field processing. Computer Graphics Forum, 32(5):73-82, 2013

[3] O. Azencot, M. Ovsjanikov, F. Chazal, and M. Ben-Chen. Discrete derivatives of vector fields on surfaces-an operator approach. ACM Transactions on Graphics, 34(3):Article No. 29, 2015.

[4] I. Baeza Rojo and T. Günther. Vector field topology of time-dependent flows in a steady reference frame. IEEE Transactions on Visualization and Computer Graphics, 26(1):280-290, 2020.

[5] D. C. Banks and B. A. Singer. A predictor-corrector technique for visualizing unsteady flow. IEEE Transactions on Visualization and Computer Graphics, 1(2):151-163, 1995.

[6] M. Ben-Chen, A. Butscher, J. Solomon, and L. Guibas. On discrete Killing vector fields and patterns on surfaces. In Proceedings of Eurographics Symposium on Geometry Processing, pp. 1701-1711, 2010.

[7] R. Bujack, M. Hlawitschka, and K. I. Joy. Topology-inspired Galilean invariant vector field analysis. In Proceedings of IEEE Pacific Visualization 2016, pp. 72-79, 2016

[8] B. Cabral and L. C. Leedom. Imaging vector fields using line integral convolution. In Proceedings of SIGGRAPH '93, pp. 263-270, 1993.

[9] M. P. do Carmo. Riemannian Geometry. Birkhäuser, 1992.

[10] E. Dresselhaus and M. Tabor. The kinematics of stretching and alignment of material elements in general flow fields. Journal of Fluid Mechanics, 236:415-444, 1991.

[11] R. Drouot and M. Lucius. Approximation du second ordre de la loi de comportement des fluides simples. Lois classiques déduites de l'introduction d'un nouveau tenseur objectif. Archiwum Mechaniki Stosowanej, 28(2):189-198, 1976.

[12] T. Frankel. The Geometry of Physics: An Introduction. Cambridge University Press, 3rd ed., 2011

[13] C. Garth, X. Tricoche, T. Salzbrunn, T. Bobach, and G. Scheuermann. Surface techniques for vortex visualization. In Proceedings of Eurograph ics/IEEE VGTC Symposium on Visualization, pp. 155-164, 2004.

[14] T. Günther, M. Gross, and H. Theisel. Generic objective vortices for flow visualization. ACM Transactions on Graphics, 36(4):Article No. 141, 2017.

[15] T. Günther, M. Schulze, and H. Theisel. Rotation invariant vortices for flow visualization. IEEE Transactions on Visualization and Computer Graphics, 22(1):817-826, 2016.

[16] T. Günther and H. Theisel. Hyper-objective vortices. IEEE Transactions on Visualization and Computer Graphics, 25(1):1-1, 2018.

[17] T. Günther and H. Theisel. The State of the Art in Vortex Extraction. Computer Graphics Forum, 37(6):149-173, 2018.

[18] M. Hadwiger, M. Mlejnek, T. Theuß1, and P. Rautek. Time-dependent flow seen through approximate observer Killing fields. IEEE Transactions on Visualization and Computer Graphics, 25(1):1257-1266, 2019.

[19] G. Haller. An objective definition of a vortex. Journal of Fluid Mechanics, 525:1-26, 2005.

[20] G. Haller. Can vortex criteria be objectivized? Journal of Fluid Mechanics, 508:A25, 2021.

[21] G. Haller, A. Hadjighasem, M. Farazmand, and F. Huhn. Defining coherent vortices objectively from the vorticity. Journal of Fluid Mechanics, 795:136-173, 2016

[22] G. A. Holzapfel. Nonlinear Solid Mechanics: A Continuum Approach for Engineering. Wiley, 2000.

[23] J. C. R. Hunt, A. A. Wray, and P. Moin. Eddies, streams, and convergence zones in turbulent flows. In Proceedings of the Summer Program 1988 pp. 193-208. Center for Turbulence Research, Dec. 1988.

[24] J. Jeong and F. Hussain. On the identification of a vortex. Journal of Fluid Mechanics, 285:69-94, 1995.

[25] B. Jobard, G. Erlebacher, and M. Y. Hussaini. Lagrangian-Eulerian advection of noise and dye textures for unsteady flow visualization. IEEE Transactions on Visualization and Computer Graphics, 8(3):211-222, 2002.

[26] B. Jobard and W. Lefer. Creating evenly-spaced streamlines of arbitrary density. In 8th Eurographics Workshop on Visualization in Scientific Computing, pp. 211-222, 1997.

[27] C. Jung, T. Tél, and E. Ziemniak. Application of scattering chaos to particle transport in a hydrodynamical flow. Chaos: An Interdisciplinary
Journal of Nonlinear Science, 3(4):555-568, 1993.

[28] M. Kilian, N. J. Mitra, and H. Pottmann. Geometric modeling in shape space. ACM Transactions on Graphics, 26(3):Article No. 64, 2007.

[29] H. J. Lugt. The dilemma of defining a vortex. In Recent Developments in Theoretical and Experimental Fluid Mechanics: Compressible and Incompressible Flows, pp. 309-321. Springer-Verlag, 1979.

[30] J. E. Marsden and T. J. Hughes. Mathematical Foundations of Elasticity. Dover Publications, Inc., 1994.

[31] J. Martinez Esturo, C. Rössl, and H. Theisel. Generalized metric energies for continuous shape deformation. In Mathematical Methods for Curves and Surfaces, Lecture Notes in Computer Science, pp. 135-157. SpringerVerlag, 2012

[32] A. McInerney. First Steps in Differential Geometry. Springer-Verlag, 2013.

[33] R. W. Ogden. Non-Linear Elastic Deformations. Dover Publications, Inc., 1997.

[34] A. Okubo. Horizontal dispersion of floatable particles in the vicinity of velocity singularities such as convergences. Deep Sea Research and Oceanographic Abstracts, 17(3):445-454, June 1970.

[35] R. Peikert and M. Roth. The "Parallel Vectors" operator-a vector field visualization primitive. In Proceedings of IEEE Visualization '99, pp. 263-532, 1999

[36] A. E. Perry and M. S. Chong. A description of eddying motions and flow patterns using critical-point concepts. Ann. Rev. Fluid Mech., 19(1):125$155,1987$.

[37] A. E. Perry and M. S. Chong. Topology of flow patterns in vortex motions and turbulence. Applied Scientific Research, 53(3):357-374, 1994

[38] P. Petersen. Riemannian Geometry. Springer-Verlag, 3rd ed., 2016.

[39] P. Rautek, M. Mlejnek, J. Beyer, J. Troidl, H. Pfister, T. Theuß1, and M. Hadwiger. Objective observer-relative flow visualization in curved spaces for unsteady $2 \mathrm{~d}$ geophysical flows. IEEE Transactions on Visualization and Computer Graphics, 27(2):283-293, 2021.

[40] S. K. Robinson. A review of vortex structures and associated coherent motions in turbulent boundary layers. In Proceedings of Structure of Turbulence and Drag Reduction, IUTAM Symposium, pp. 23-50. SpringerVerlag, 1990.

[41] P. G. Saffman. Vortex Dynamics. Cambridge University Press, 1995.

[42] J. Sahner, T. Weinkauf, and H.-C. Hege. Galilean invariant extraction and iconic representation of vortex core lines. In Proceedings of Eurographics/IEEE VGTC Symposium on Visualization, pp. 151-160, 2005.

[43] J. Solomon, M. Ben-Chen, A. Butscher, and L. Guibas. As-Killing-aspossible vector fields for planar deformation. In Proceedings of Eurographics Symposium on Geometry Processing, pp. 1543-1552, 2011.

[44] D. Sujudi and R. Haimes. Identification of swirling flow in 3-d vector fields. In Proceedings of the 12th Computational Fluid Dynamics Conference, pp. 792-799, 1995.

[45] H. Theisel, M. Hadwiger, P. Rautek, T. Theuß1, and T. Günther. Vortex criteria can be objectivized by unsteadiness minimization. arXiv:2106.16169 [physics.flu-dyn], 2021.

[46] C. Truesdell and W. Noll. The Nonlinear Field Theories of Mechanics. Springer-Verlag, 1965.

[47] J. J. van Wijk. Image based flow visualization. ACM Transactions on Graphics, 21(3):745-754, 2002.

[48] T. Weinkauf, J. Sahner, H. Theisel, and H.-C. Hege. Cores of swirling particle motion in unsteady flows. IEEE Transactions on Visualization and Computer Graphics, 13(6):1759-1766, 2007.

[49] T. Weinkauf and H. Theisel. Streak lines as tangent curves of a derived vector field. IEEE Transactions on Visualization and Computer Graphics, 16(6): 1225-1234, 2010.

[50] J. Weiss. The dynamics of enstrophy transfer in two-dimensional hydrodynamics. Physica D: Nonlinear Phenomena, 48(2):273-294, Mar. 1991.

[51] A. Wiebel, R. Chan, C. Wolf, A. Robitzki, A. Stevens, and G. Scheuermann. Topological flow structures in a mathematical model for rotationmediated cell aggregation. In Topological Methods in Data Analysis and Visualization, Mathematics and Visualization, pp. 193-204, 2009. 